\title{
BRAF V600E mutation and BRAF VE1 immunoexpression profiles in different types of glioblastoma
}

\author{
ZEYNEP TOSUNER ${ }^{1}$, MELIN ÖZGÜN GEÇER ${ }^{1}$, MUSTAFA AZIZ HATIBOĞLU ${ }^{2}$, \\ ANAS ABDALLAH $^{2}$ and SEVAL TURNA ${ }^{1}$
}

Departments of ${ }^{1}$ Pathology and ${ }^{2}$ Neurosurgery, Faculty of Medicine, Bezmialem Vakıf University, İstanbul 34093, Turkey

Received November 26, 2017; Accepted April 27, 2018

DOI: $10.3892 / \mathrm{ol} .2018 .8919$

\begin{abstract}
Abnormalities in proto-oncogene B-Raf (BRAF) are typical in several subgroups of gliomas, including pilocytic astrocytomas, optic nerve gliomas, pleomorphic xanthoastrocytomas (PXA), anaplastic PXAs and gangliogliomas. However, they are rarely reported in adult gliomas. BRAF alterations are frequent in a distinct variant of glioblastomas (GBMs) known as epithelioid GBMs (E-GBMs). There are limited studies on whether immunohistochemistry (IHC) can be used to determine the presence of BRAF VE1 mutations in these tumors. The aim of the current study was to examine BRAF V600E mutations in 20 GBMs, including GBMs with epithelioid features, giant cell GBMs and conventional GBMs. V600 mutations were detected using the Cobas 4800 BRAF V600 Mutation Test, and IHC analysis was also performed. Of the 6 cases of GBM with epithelioid features, 1 exhibited a BRAF V600E mutation, while the other cases did not. IHC staining was positive in 3 out of the 8 conventional GBMs. Vemurafenib is a targeted therapy that has mainly been used for the treatment of melanoma patients for several years, and as a possible alternative treatment for cases of GBM harboring BRAF mutations, its existence may make testing for BRAF status important.
\end{abstract}

\section{Introduction}

Advances in genomic research have revealed various mutations in cancer-associated genes. One of these mutations is located in the proto-oncogene B-Raf (BRAF) gene, and has been detected in a wide range of cancer types, including malignant melanoma, papillary thyroid carcinoma and colorectal cancer (1).

Correspondence to: Dr Zeynep Tosuner, Department of Pathology, Faculty of Medicine, Bezmialem Vakıf University, Adnan Menderes Boulevard, Vatan Street, Fatih, İstanbul 34093, Turkey

E-mail: zeyneptosuner@yahoo.com.tr

Key words: BRAF V600E, BRAF VE1, glioblastoma, epithelioid glioblastoma
BRAF is a serine/threonine kinase and a member of the RAF family, which consists of three kinases: ARAF, CRAF (RAF-1) and BRAF. BRAF is a key regulator of the mitogen-activated protein kinase/extracellular signal-regulated kinase pathway (2). Hyperactivation of this pathway can lead to cell cycle arrest, while aberrant regulation of the pathway can trigger carcinogenesis (1).

In total, $>40$ mutations have been identified in the BRAF gene, and $90 \%$ of these are accounted for by a single base change of thymine-to-adenine at position 1,799. This missense mutation in exon 15 results in a change at residue 600, where glutamine is substituted for valine (V600E). BRAF V600E can lead to a 500-fold increase in activation of the gene. Additionally, it also permits signaling cascade activation in the absence of any extracellular stimuli, such as growth signals, and the cells can therefore become self-sufficient within this pathway (2).

The BRAF V600E mutation is detected in $4 \%$ of primary and metastatic central nervous system neoplasms. Among metastatic cases, melanoma, papillary thyroid carcinoma and hepatocellular carcinoma cerebral metastases have been found to possess this mutation (3).

Point or fusion mutations in BRAF have also been identified in glial and glioneuronal tumors, particularly in pediatric-onset cases. The majority of BRAF mutations are BRAF V600 mutations. BRAF V600E frequently occurs in pleomorphic xanthoastrocytoma (PXA), ganglioglioma and extra-cerebellar pilocytic astrocytoma (4). BRAF V600E is also detected in $1 \%$ of primary brain tumors, including glioblastoma (GBM), gliosarcoma, diffuse astrocytoma and rhabdoid meningioma (3).

Epithelioid GBM (E-GBM) is a novel entity according to the World Health Organization (WHO) 2016 brain tumor classification (5). The composition of an E-GBM is based on cohesive sheets of closely-packed, patternless, variably lipidized, small-to medium-sized cells that have rounded cytoplasmic borders, an eosinophilic cytoplasm and lack any cytoplasmic stellate processes, with interspersed neuropils (6-11). The tumors also harbor BRAF V600E mutations $(3,11,12)$.

Anti-BRAF V600E (clone VE1), a mouse monoclonal primary antibody [anti-BRAF V600E (VE1)] is used in the identification of the mutant BRAF V600E protein. BRAF VE1 has been successfully validated in malignant melanoma, 
colorectal carcinoma, papillary thyroid carcinoma, lung cancer and PXA (13-19).

The present study aimed to investigate the presence of the BRAF V600E mutation and the immunoexpression profiles of different types of GBMs treated at Bezmialem Vak1f University (İstanbul, Turkey), and to determine the efficiency of the BRAF mutation-specific VE1 antibody to detect the V600E hotspot mutation successfully. In addition, the potential associations between the clinical parameters of these cases and BRAF status were analyzed.

\section{Materials and methods}

Patients and tumor classification. A total of 20 patients with GBM between January 2015 and January 2017 were included in this study in order to analyze the immunoexpression of BRAF (BRAF VE1) and the BRAF V600E mutation. Newly diagnosed glioblastomas were included in the study. Exclusion criteria were prior chemotherapy, antiangiogenic therapy and radiotherapy.

This study was approved by the Ethics Committee of Bezmialem Vakıf University Faculty of Medicine (İstanbul, Turkey). Written informed consent was obtained from all patients included in this study. The expression levels of these antigens and their associations with the clinical follow-up data, including the patient's age, sex and overall survival status, were assessed.

Clinical information and follow-up data were obtained from the Department of Neurosurgery. Telephone interviews of the patients or family members were performed for the study. Overall survival was defined from the time of diagnosis to mortality from any cause of death.

All hematoxylin and eosin-stained slides were reviewed by two pathologists for the confirmation of the diagnoses. GBMs were considered as having epithelioid features when $>30 \%$ of the tumor was composed of epithelioid cells. Tumors with a predominance of bizarre, multinucleated giant cells and occasional abundant stromal reticulin networks were considered as giant cell GBMs. Tumors were further categorized into two types for statistical analysis: GBMs with epithelioid features and GBMs with non-epithelioid features.

Mutation analysis. Samples from all 20 patients were analyzed for V600 mutations using the Cobas 4800 BRAF V600 Mutation Test (Roche Molecular Diagnostics, Pleasanton CA, USA), which is a European Conformity (CE)-marked and Food and Drug Administration (FDA)-approved in vitro diagnostics device designed to detect the BRAF p.Val600Glu mutation in DNA derived from formalin-fixed, paraffin-embedded (FFPE) melanoma samples. The method consisted of a quantitative polymerase chain reaction (PCR) step with two primers that amplified a 116-bp fragment of exon 15 of BRAF (containing codon 600), and two hydrolysis probes labeled with different fluorochromes [one for the wild-type (WT) allele and one for the mutant allele, p.Val600Glu and a quencher]. The quencher absorbed the light from the reporter dye until the DNA polymerase cleaved it with its 5'-3' exonuclease activity during the amplification steps. Once the reporter was free, it emitted fluorescence at a specific wavelength, which differed for the WT and p.Val600Glu alleles. The amount of light detected throughout the PCR for each dye was directly proportional to the original amount of WT or p.Val600Glu template; therefore, at the end of the process, the software calculated the ratio of these two signals to report each sample as 'mutation detected' or 'mutation not detected'. Selective amplification was achieved by using uracil-N-glycosylase and dUTP in the reaction mix to thereby eliminate any potential contamination during the first step of the reaction prior to PCR amplification. The kit also contained a WT and a p.Val600Glu control tube that were analyzed in each run to ensure performance of the test. The Cobas z480 (Roche Molecular Diagnostics) (offered software for automated analysis and interpretation of the results. The analysis was performed according to the manufacturer's protocol.

Immunohistochemistry (IHC). Sections (4- $\mu$ m thick) of FFPE tissues were placed on 3-aminopropyletxylene-covered slides. Subsequently, they were stained with mouse monoclonal antibody against BRAF V600E (catalog no. 790-4855; 1/100 titer; clone VE1) following the Ventana Medical Systems' protocol. Briefly, staining was performed on a Ventana BenchMark Ultra (Ventana Medical Systems, Inc., Tucson, AZ, USA). The staining protocol included use of Cell Conditioning 1 (Ventana Medical Systems, Inc.) for $64 \mathrm{~min}$, pre-peroxidase inhibition with $3 \%$ hydrogen peroxide for $10 \mathrm{~min}$ at $37^{\circ} \mathrm{C}$, primary antibody incubation for $70 \mathrm{~min}$ and Amplification kit (catalog no. 760-080; Ventana Medical Inc.) was applied for 4 min at $37^{\circ} \mathrm{C}$ to increase the signal intensity. The OptiView DAB IHC Detection kit (Ventana Medical Systems, Inc.) was used to detect BRAF V600E protein expression. Tissues were counterstained with hematoxylin for $16 \mathrm{~min}$ and Bluing Reagent for $4 \mathrm{~min}$. Counterstaining was applied at room temperature. After the procedure the slides were reviewed using Nikon Ci-E microscope (Nikon Corporation, Tokyo, Japan) at x40, x100, x200 and x400 magnifications.

All of the cases were evaluated with an immunohistochemical panel consisting of glial fibrillary acidic protein (GFAP), Olig2, epithelial membrane antigene (EMA), CK (Pancytokeratin) and Ki-67 antibodies at time of initial diagnosis. The staining was also performed on a Ventana BenchMark Ultra (Ventana Medical Systems, Inc., Tucson, AZ, USA) with immunostaining protocols for GFAP (catalog no. RB-087-R7; Thermo Fisher Scientific, Inc., Waltham, MA, USA) 1:100 dilution for $20 \mathrm{~min}$, Olig2 (catalog no. GTX31569; GeneTex, Irvine, CA, USA) 1:100 dilution for $20 \mathrm{~min}$, EMA (catalog no. MS-348-R7; Thermo Fisher Scientific, Inc.) 1:200 dilution for $30 \mathrm{~min}, \mathrm{CK}$ (Keratin Clone AE1/AE3, catalog no. MS-343-R7; Thermo Fisher Scientific, Inc.) 1:50 for 20 min, Vimentin (catalog no. MS-129-R7; Thermo Fisher Scientific, Inc. ) 1:50 dilution for $20 \mathrm{~min}$ and Ki67 (catalog no. RB-1510-R7; Thermo Fisher Scientific, Inc.) 1:100 dilution for $30 \mathrm{~min}$ at room temperature.

Evaluation of immunostaining. Immunostaining with the BRAF VE-1 antibody was evaluated in 10 representative fields. The cases were considered as positive when diffuse near-complete cytoplasmic and membranous staining was visible at scanning magnification. Background staining and staining in non-tumoral cells was considered as non-specific staining, and cases with this kind of staining pattern were 
Table I. Summary of clinical, morphological and molecular data of the patients.

\begin{tabular}{|c|c|c|c|c|c|c|c|c|}
\hline $\begin{array}{l}\text { Patient } \\
\text { no. }\end{array}$ & $\begin{array}{l}\text { Age, } \\
\text { years }\end{array}$ & Sex & Localization & Morphology & $\begin{array}{l}\text { BRAF } \\
\text { IHC }\end{array}$ & $\begin{array}{c}\text { BRAF V600E } \\
\text { mutation }\end{array}$ & $\begin{array}{l}\text { Survival } \\
\text { time, days }\end{array}$ & $\begin{array}{l}\text { Survival } \\
\text { status }\end{array}$ \\
\hline 1 & 44 & M & Parietal & $\mathrm{E}$ & - & + & 304 & Alive \\
\hline 2 & 2 & M & Frontotemporal & $\mathrm{E}$ & - & - & 185 & Succumbed \\
\hline 3 & 17 & $\mathrm{~F}$ & Parietal & $\mathrm{E}$ & - & - & NA & NA \\
\hline 4 & 49 & M & Frontal & $\mathrm{E}$ & - & - & 396 & Alive \\
\hline 5 & 17 & $\mathrm{~F}$ & Parietal & $\mathrm{E}$ & - & - & 211 & Alive \\
\hline 6 & 74 & $\mathrm{M}$ & Temporal & $\mathrm{E}$ & - & - & NA & NA \\
\hline 7 & 73 & M & Frontoparietal & $\mathrm{GC}$ & - & - & 132 & Succumbed \\
\hline 8 & 71 & M & Occipital & $\mathrm{GC}$ & - & - & 541 & Succumbed \\
\hline 9 & 62 & $\mathrm{~F}$ & Frontal & $\mathrm{GC}$ & - & - & NA & NA \\
\hline 10 & 30 & $\mathrm{~F}$ & Frontal & $\mathrm{GC}$ & - & - & 710 & Alive \\
\hline 11 & 46 & $\mathrm{~F}$ & Frontal & $\mathrm{GC}$ & - & - & 827 & Alive \\
\hline 12 & 53 & $\mathrm{~F}$ & Frontoparietal & $\mathrm{GC}$ & - & - & 13 & Succumbed \\
\hline 13 & 53 & M & Frontoparietal & $\mathrm{C}$ & - & - & 251 & Succumbed \\
\hline 14 & 80 & $\mathrm{M}$ & Frontotemporal & $\mathrm{C}$ & - & - & 268 & Succumbed \\
\hline 15 & 71 & $\mathrm{~F}$ & Temporal & $\mathrm{C}$ & + & - & 536 & Alive \\
\hline 16 & 61 & $\mathrm{M}$ & Frontal & $\mathrm{C}$ & + & - & 360 & Succumbed \\
\hline 17 & 13 & $\mathrm{M}$ & Temporal & $\mathrm{C}$ & + & - & 276 & Alive \\
\hline 18 & 55 & M & Temporal & $\mathrm{C}$ & - & - & 166 & Succumbed \\
\hline 19 & 65 & $\mathrm{~F}$ & Frontal & $\mathrm{C}$ & - & - & NA & NA \\
\hline 20 & 69 & $\mathrm{M}$ & Frontoparietal & $\mathrm{C}$ & - & - & NA & NA \\
\hline
\end{tabular}

M, male; F, female; E, glioblastomas with epithelioid features; GC, giant cell glioblastomas; C, conventional; IHC, immunohistochemistry; NA, not assessed; BRAF, proto-oncogene B-Raf.

interpreted as negative. A PE block prepared from a case of papillary thyroid carcinoma showing diffuse intense staining with the BRAF VE1 antibody was also used as an external control tissue.

Statistical analysis. Statistical analysis was performed using Number Cruncher Statistical System 2007 (NCSS; LLC, Kaysville, UT, USA). Quantitative statistics (mean, standard deviation and frequency) and qualitative data were analyzed. Normally distributed parameters between groups were analyzed with the unpaired Student's t-test and the unevenly distributed data were analyzed using the Mann Whitney U-test. Frequency data were assessed with the $\chi^{2}$ test or the Fisher's exact test. Survival was evaluated with Kaplan-Meier survival analysis excluding those lost to follow-up and who succumbed due to postoperative surgical complications. Log-rank (Mantel-Cox) analysis was used to compare the prognostic significance of the antibody staining level for survival. Results were presented with $95 \%$ confidence intervals, and $\mathrm{P}<0.05$ was considered to indicate a statistically significant difference.

\section{Results}

Patients and follow-up. A total of 20 patients (12 men and 8 women) were included in this study. The age of the patients ranged from 2 to 80 years, and the median age at time of diagnosis was 54 years. The tumors were parietal $(n=3)$, frontotemporal $(n=2)$, frontal $(n=6)$, temporal $(n=4)$, frontoparietal $(n=4)$ and occipital $(n=1)$.

Data from clinical follow-up of up to 827 days was available for 15 out of 20 patients. The mean survival time was 464.0 days (range, 13-827 days). The overall mortality rate of the patients with GBM was $53.3 \%$ (8 of the 15 patients).

Patients with E-GBM ranged in age from 2-74 years. Of the 6 patients with E-GBM, 5 patients were $<50$ years old, and 3 were pediatric ( $\leq 18$ years old). Of the pediatric patients, 1 patient was 2 years old and the others were 17 years old at the time of diagnosis. In the non-epithelioid GBM (NE-GBM) group, the majority of the patients were aged $>50$ years. Only 1 patient with conventional GBM was pediatric (13 years old) (Table I).

The associations between age, sex and localization between the two histological types [E-GBM and NE-GBM) were analyzed. No significant difference was identified between the two groups for these parameters $(\mathrm{P}>0.05)$. The estimated mean survival times were 434 and 343 days in NE-GBMs and E-GBMs, respectively. Although the survival time was shorter in E-GBMs, there was no statistically significant difference (P>0.05) Kaplan Meier Analysis, $\chi^{2}$ Test, Log Rank (Mantel Cox) (Table II).

Histopathological evaluation. According to the WHO classification, 8 tumors were of the conventional type, 6 were giant cell GBMs and 6 had epithelioid features. 
Table II. Mean survival times (days).

\begin{tabular}{lcccc}
\hline & & \multicolumn{3}{c}{ Mean } \\
\cline { 3 - 5 } & & \multicolumn{3}{c}{$95 \%$ Confidence interval } \\
\cline { 3 - 5 } Morphology & Estimate $^{\mathrm{a}}$ & Std Error & Lower limit & Upper limit \\
\hline NE-GBMs & 434,000 & 90,878 & 255,879 & 612,121 \\
E-GBMS & 343,250 & 45,683 & 253,712 & 432,788 \\
All GBMs & 464,030 & 82,945 & 301,459 & 626,602 \\
\hline
\end{tabular}

( $\mathrm{P}>0.05)$ Kaplan Meier Analysis, $\chi^{2}$-test. ${ }^{a}$ Estimation is limited to the largest survival time if it is censored. E-GBM, epithelioid GBM; NE, non-E-GBM.

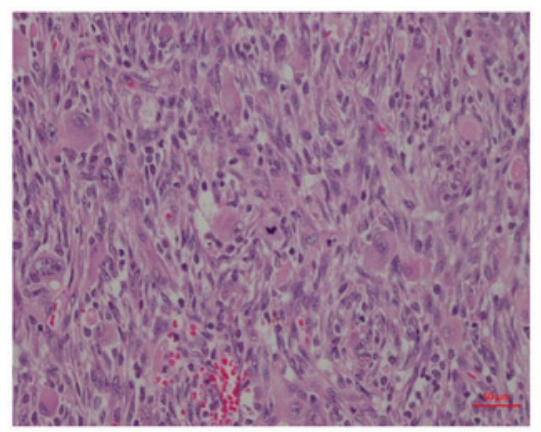

Figure 1. A case of epithelioid glioblastoma composed of epithelioid discohesive cells with eosinophilic round cytoplasm and nuclei with distinct eccentrically nucleoli (hematoxylin and eosin staining; x200 magnification).

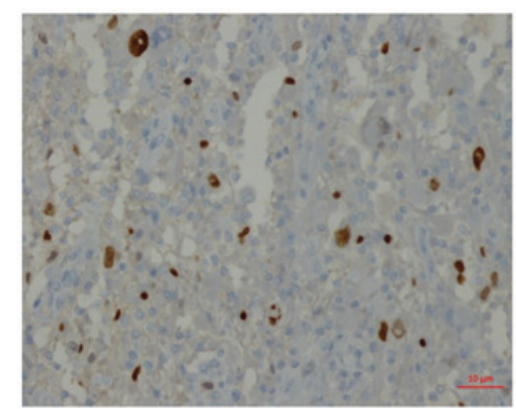

Figure 2. Focal immunostaining with oligodendrocyte transcription factor in epithelioid glioblastoma (x200 magnification).

Conventional and giant cell GBMs were considered as NE-GBMs. The mortality rates were $25 \%$ in E-GBMs and $63.6 \%$ in NE-GBMs.

E-GBMs were composed of epithelioid cells with eosinophilic round cytoplasm devoid of stellate cytoplasmic processes, and exhibited nuclei with distinct nucleoli that were eccentrically located. These cells were discohesive and arranged in patternless and closely packed sheets (Fig. 1). A number of the cells had intranuclear inclusions. All of the E-GBM cases showed microvascular proliferation and palisading/confluent necrosis. None of the E-GBMs showed eosinophilic granular bodies, which are a distinct feature of PXAs. Mitotic activity was also high in the E-GBM cases [>7 per 10 high-power fields

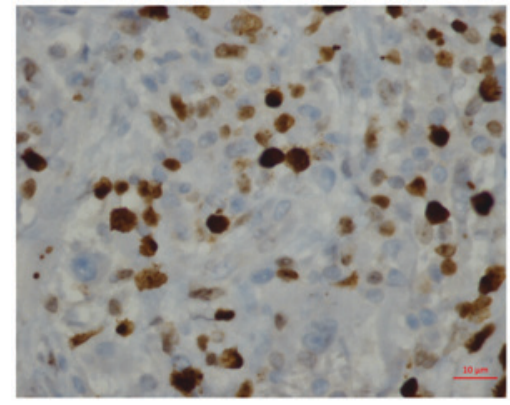

Figure 3. Ki-67 immunostaining in a case of epithelioid glioblastoma, with $40 \%$ activity (x400 magnification).

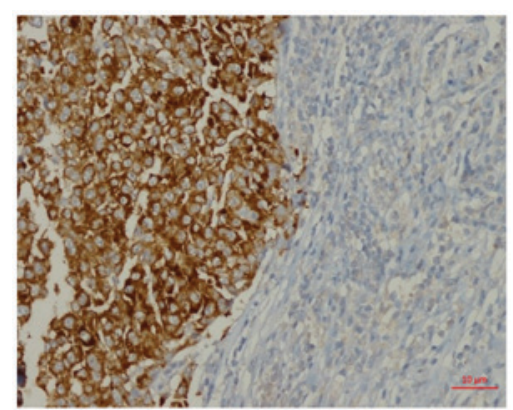

Figure 4. Proto-oncogene B-Raf VE1 immunostaining in a papillary thyroid carcinoma case used as the external positive control tissue (x400 magnification).

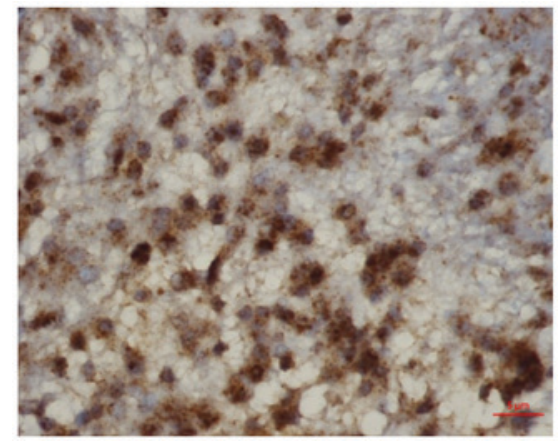

Figure 5. Diffuse and intense proto-oncogene B-Raf VE1 immunostaining in a case of conventional glioblastoma (x400 magnification).

(HPFs) in each case]. In 2 cases, 40 mitoses per 10 HPFs were recorded, and the mean mitotic activity was 23 per 10 HPFs. IHC staining in the 6 cases showed focal and/or weak staining with GFAP and focal nuclear staining with oligodendrocyte transcription factor (Olig2) (Fig. 2). Focal immunoexpression with epithelial markers, such as cytokeratin and epithelial membrane antigen, was present in each E-GBM case. The Ki-67 proliferation index was also high in these cases. The lowest Ki-67 index was $20 \%$, the highest was $55 \%$ and the mean was $40 \%$ (Fig. 3).

NE-GBMs were either conventional or giant cell GBMs. Conventional GBMs were composed of neoplastic glial cells, the majority of which had distinct stellate cytoplasmic processes, and all were intensely immunostained with GFAP and Olig2. Giant cell GBMs had a $>50 \%$ giant cell component throughout the whole tumor. The giant cells were bizarre and 
multinucleated. All the giant cell GBMs had an abundant stromal reticulin network and had glial regions, stained with GFAP, and sarcomatous regions, stained with vimentin. The mean mitotic activity of the NE-GBMs was 15 per 10 HPFs and the mean Ki-67 index was $37 \%$.

Immunoexpression of BRAF VE1 and BRAF V600E mutation. Among the conventional GBMs, 3 showed positive immunostaining with the BRAF VE1 antibody, while no immunoreactivity was observed in the other cases (Figs. 4 and 5). Diffuse intense cytoplasmic staining, mild membranous staining and a certain degree of granular staining were present in the positive cases. Only 1 E-GBM patient (a 44-year-old male) exhibited the BRAF V600E mutation. In this patient, the tumor was located in the left parietal lobe, and magnetic resonance imaging results indicated possible metastasis. The mitotic count was 11 per $10 \mathrm{HPFs}$ and the Ki-67 proliferation index was 55\%. BRAF VE1 immunoexpression was not observed in this case. IHC testing was repeated, but the second test invariably showed no staining. The association between BRAF immunohistochemistry and BRAF mutation was analysed using Fisher's Exact Test. No statistical association was identified between mutation and immunohistochemistry $(\mathrm{P}>0.05)$.

The BRAF V600E mutation ratio was $16.6 \%$ in E-GBMs and $5 \%$ in the total 20 cases of GBM included in the study. The relation between clinical parameters such as age, sex and localization of the patients was analysed using Fisher's Exact Test. No statistically significant associations were found between the clinical parameters and the BRAF status of the tumors $(\mathrm{P}>0.05)$.

\section{Discussion}

The present study demonstrated that $16.7 \%$ of E-GBMs (1/6 cases) possessed the BRAF V600E mutation. BRAF V600E mutations are relatively common in PXAs (43-66\%), anaplastic PXAs (65\%) and gangliogliomas (18-43\%), whereas they are more rare in adult gliomas (4,20-22). Approximately $22 \%$ of gliosarcomas possess a BRAF mutation (23). Kleinschmidt-DeMasters et al (12) found that $53.8 \%$ of E-GBMs (7/13 cases) exhibited a BRAF V600E mutation.

E-GBMs and PXAs with anaplastically transformed foci (A-PXAs) show overlapping features, such as epithelioid foci with the presence of large melanoma-like tumor cells with abundant eosinophilic cytoplasm and nuclei that are occasionally eccentrically located. The two tumor types show focal epithelial and glial marker expression, retained integrase interactor 1 and Brahma-related gene 1 expression, occasional positivity for cluster of differentiation 34 and a lack of immunoreactivity for mutant isocitrate dehydrogenase 1 (R132H), and may have BRAF V600E mutations. Due to the common histological, immunohistochemical, molecular and clinical features of E-GBMs and anaplastic PXAs, one study suggested that they are closely associated or are even the same entity (24). However, unlike A-PXAs, eosinophilic granular bodies are not a feature of E-GBMs. A-PXAs show low-grade regions with more cytologically uniform cells, while E-GBMs are composed of relatively monotonous, epithelioid cells in large regions (25).
Tanaka et al (26) also reported an E-GBM with the BRAF V600E mutation arising from PXA 13 years after the treatment of the tumor. The findings in this unusual case suggested the possibility of PXA being a precursor of E-GBM.

A high proportion of pathologists and neuropathologists diagnose E-GBMs as separate entities $(6-10,25)$. E-GBMs were also introduced as a separate entity in the WHO 2016 Central Nervous System Classification guidelines (5). None of the E-GBMs in the present study had eosinophilic granular bodies or low-grade areas, which are observed in A-PXAs.

Within the E-GBM cohort $(n=6)$, the patient ages ranged from 2 to 74 years, with $3 / 6$ patients $(50 \%)$ being pediatric. E-GBMs are typically detected within the first three decades of life (12,27). Behling et al (3) reported 7 cases with BRAF V600E mutations among 784 cases of primary brain tumors, and 3 of the 7 cases with BRAF V600E mutations were E-GBMs. Among these cases, 2 patients were aged $<30$ years (3).

In a study by Dahiya et al (28), the BRAF V600E mutation was investigated using the BRAF VE1 antibody in pediatric and adult cases of glioma and GBM. BRAF VE1 immunoreactivity was detected in 3 out of 25 pediatric GBMs (12.0\%) and 3 out of 39 adult GBMs (7.7\%). Of the 3 adult GBMs, 2 were giant cell variants, and mutant BRAF expression was limited to the giant cells. BRAF mutant tumors were more commonly detected in younger patients, with a mean age of 39 years (28).

In another study, next-generation sequencing and BRAF VE1 IHC were performed to detect the BRAF V600E mutation in 11 primary and 2 secondary adult GBMs, among which 1 conventional GBM exhibited the mutation. The patient was a 49-year-old man with a huge multicystic mass in the right occipitoparietal area. Histopathologically, epithelioid or ribbon-like epithelial structures were not present (29).

One of the aims of the current study was to evaluate whether IHC results were associated with the mutational analysis results. IHC staining with the BRAF VE1 antibody was negative in the only BRAF V600E mutated E-GBM case, and three immunopositive conventional GBM cases did not harbor the mutation.

IHC is a fast and easy method for the detection of mutations. However, optimization of IHC depends on several factors, including tissue preservation, fixation, endogenous peroxidase activity, temperature, and primary and secondary antibody concentrations. For the BRAF V600E mutant case, a repeat IHC was performed, which showed the same negative result, whereas the external control tissue was intensely stained. The exact explanations for the false-negative and false-positive results of these cases are unknown, but PCR failure can be excluded, as all samples consisted of viable adequate tumor tissues for reliable examination.

In a study by Kleinschmidt-DeMasters et al (11), in which the BRAF V600E mutation was observed in $46 \%$ of cases, a 1:1 correlation was found between BRAF V600E mutational results and IHC results. However, limitations were indicated to exist in the immunostaining even subsequent to multiple attempts to optimize the staining technique parameters, and heavy background staining resulted in equivocal results in certain cases. 
Behling et al (3) performed BRAF VE1 immunostaining in metastatic and primary brain tumors with the same antibody clone and immunostaining apparatus used in the present study, and it was necessary to repeat the immunostaining process in 12 cases due to various factors, including insufficient staining or non-specific background staining. In that study, immunostaining was followed by Sanger sequencing for the verification of a BRAF V600E mutation, and Sanger sequencing revealed 6 false-immunopositive cases that were not mutated. The false-positive cases were breast cancer and non-small cell lung cancer metastases (3). Previously, pituitary adenomas have also been demonstrated to exhibit false-positive BRAF VE1 staining (30).

In metastatic melanoma, although genomic assays are the gold standard, the sensitivity and specificity of BRAF VE1 for determining the presence of a BRAF V600 mutation are $>94 \%$, and the use of BRAF IHC followed by genomic assays in patients with negative IHC results for BRAF is recommended in order to increase the patient number with detected BRAF mutations compared with that using either assay alone (19). To understand the reliability of BRAF VE1 IHC analyses, studies with a greater number of cases should be performed.

In the present study, the mean estimated survival time was 343 days in E-GBMs, and this was 101 days shorter than the median survival time of the NE-GBMs. E-GBMs are considered to be an aggressive variant of GBM, with early complications and short survival times (median, 169 days) (27). Nevertheless, long survival times of up to 10 years have also been reported in a subset of studies $(12,24,28,29)$. However, the number of cases available in the literature is too small to provide meaningful data on survival at present.

PLX4032 (vemurafenib), an FDA-approved kinase inhibitor, has been used effectively for the targeted treatment of metastatic melanoma $(31,32)$. Marked responses to BRAF inhibitors have also been previously reported in V600E mutant PXAs, a brainstem ganglioglioma and a pediatric GBM (24,33-36). The patient with the BRAF V600E mutation in the present study received near-total resection followed by radiotherapy with concomitant chemotherapy consisting of temozolamide. Unfortunately, 11 months later, magnetic resonance imaging revealed a recurrent giant tumor in the previous resection site, and the patient also developed a subscapular fibrosarcoma as a secondary malignancy. The status of the patient was not determined to be suitable for vemurafenib therapy.

Additional studies and literature reviews will aid in improving our understanding of the molecular signature and prognosis of this newly introduced epithelioid subtype of GBM. Routine IHC staining combined with genetic testing can be performed in young patients with GBMs. A positive test result for BRAF V600E mutational status in an E-GBM case could potentially provide the patient with an alternative, targeted treatment in the form of vemurafenib.

\section{Acknowledgements}

Not applicable.

\section{Funding}

The present study was supported by the Medical Research Council of Bezmialem Vakıf University (grant no. 3.2016/8).

\section{Availability of data and materials}

All data generated or analyzed during this study are included in this published article.

\section{Authors' contributions}

Study conception and design: ZT. Acquisition of data: ZT, ST and AH. Analysis and interpretation of data: ZT, MOG, AA and AH. Drafting of the manuscript: ZT and ST. Critical revision: $\mathrm{MOG}$ and $\mathrm{AA}$.

\section{Ethics approval and consent to participate}

This study was approved by the Ethics Committee of Bezmialem Vakıf University Faculty of Medicine (İstanbul, Turkey). Written informed consent was obtained from all patients included in this study.

\section{Consent for publication}

Not applicable.

\section{Competing interests}

The authors declare that they have no competing interests.

\section{References}

1. Davies H, Bignell GR, Cox C, Stephens P, Edkins S, Clegg S, Teague J, Woffendin $\mathrm{H}$, Garnett MJ, Bottomley W, et al: Mutations of the BRAF gene in human cancer. Nature 417: 949-954, 2002

2. Cantwell-Dorris ER, O'Leary JJ and Sheils OM: BRAFV600E: Implications for carcinogenesis and molecular therapy. Mol Cancer Ther 10: 385-394, 2011.

3. Behling F, Barrantes-Freer A, Skardelly M, Nieser M, Christians A, Stockhammer F, Rohde V, Tatagiba M, Hartmann C, Stadelmann C and Schittenhelm J: Frequency of BRAF V600E mutations in 969 central nervous system neoplasms. Diagn Pathol 11: 55, 2016.

4. Schindler G, Capper D, Meyer J, Janzarik W, Omran H, Herold-Mende C, Schmieder K, Wesseling P, Mawrin C, Hasselblatt M, et al: Analysis of BRAF V600E mutation in 1,320 nervous system tumors reveals high mutation frequencies in pleomorphic xanthoastrocy toma, ganglioglioma and extra-cerebellar pilocytic astrocytoma. Acta Neuropathol 121: 397-405, 2011.

5. Louis DN, Perry A, Reifenberger G, von Deimling A, Figarella-Branger D, Cavenee WK, Ohgaki H, Wiestler OD, Kleihues P and Ellison DW: The 2016 world health organization classification of tumors of the central nervous system: A summary. Acta Neuropathol 131: 803-820, 2016.

6. Rosenblum MK, Erlandson RA and Budzilovich GN: The lipid-rich epithelioid glioblastoma. Am J Surg Pathol 15: 925-934, 1991.

7. Akimoto J, Namatame H, Haraoka J and Kudo M: Epithelioid glioblastoma: A case report. Brain Tumor Pathol 22: 21-27, 2005.

8. Rodriguez FJ, Scheithauer BW, Giannini C, Bryant SC and Jenkins RB: Epithelial and pseudoepithelial differentiation in glioblastoma and gliosarcoma: A comparative morphologic and molecular genetic study. Cancer 113: 2779-2789, 2008.

9. Gasco J, Franklin B, Fuller GN, Salinas P and Prabhu S: Multifocal epithelioid glioblastoma mimicking cerebral metastasis: Case report. Neurocirugia (Astur) 20: 550-554, 2009.

10. Tanaka S, Nakada M, Hayashi Y, Nakada S, Sawada-Kitamura S, Furuyama N, Suzuki T, Kamide T, Hayashi Y, Yano S and Hamada J: Epithelioid glioblastoma changed to typical glioblastoma: The methylation status of MGMT promoter and 5-ALA fluorescence. Brain Tumor Pathol 28: 59-64, 2011. 
11. Kleinschmidt-DeMasters BK, Aisner DL and Foreman NK: BRAF VE1 immunoreactivity patterns in epithelioid glioblastomas positive for BRAF V600E mutation. Am J Surg Pathol 39: 528-540, 2015

12. Kleinschmidt-DeMasters BK, Aisner DL, Birks DK and Foreman NK: Epithelioid GBMs show a high percentage of BRAF V600E mutation. Am J Surg Pathol 37: 685-698, 2013.

13. Capper D, Preusser M, Habel A, Sahm F, Ackermann U, Schindler G, Pusch S, Mechtersheimer G, Zentgraf H and von Deimling A: Assessment of BRAF V600E mutation status by immunohistochemistry with a mutation-specific monoclonal antibody. Acta Neuropathol 122: 11-19, 2011.

14. Colomba E, Hélias-Rodzewicz Z, Von Deimling A, Marin C, Terrones N, Pechaud D, Surel S, Côté JF, Peschaud F, Capper D, et al: Detection of BRAF p.V600E mutations in melanomas: comparison of four methods argues for sequential use of immunohistochemistry and pyrosequencing. J Mol Diagn 15: 94-100, 2013.

15. Dvorak K, Aggeler B, Palting J, McKelvie P, Ruszkiewicz A and Waring P: Immunohistochemistry with the anti-BRAF V600E (VE1) antibody: Impact of pre-analytical conditions and concordance with DNA sequencing in colorectal and papillary thyroid carcinoma. Pathology 46: 509-517, 2014.

16. Ilie $\mathrm{M}$, Long E, Hofman V, Dadone B, Marquette $\mathrm{CH}$, Mouroux J, Vignaud JM, Begueret H, Merlio JP, Capper D, et al: Diagnostic value of immunohistochemistry for the detection of the BRAFV600E mutation in primary lung adenocarcinoma Caucasian patients. Ann Oncol 24: 742-748, 2013.

17. Ida CM, Vrana JA, Rodriguez FJ, Jentoft ME, Caron AA, Jenkins SM and Giannini C: Immunohistochemistry is highly sensitive and specific for detection of BRAF V600E mutation in pleomorphic xanthoastrocytoma. Acta Neuropathol Commun 1: 20, 2013.

18. Ritterhouse LL and Barletta JA: BRAF V600E mutation-specific antibody: A review. Semin Diagn Pathol 32: 400-408, 2015.

19. Lo MC, Paterson A, Maraka J, Clark R, Goodwill J, Nobes J, Garioch J, Moncrieff M, Rytina E and Igali L: A UK feasibility and validation study of the VE1 monoclonal antibody immunohistochemistry stain for BRAF-V600E mutations in metastatic melanoma. Br J Cancer 115: 223-227, 2016.

20. Gierke M, Sperveslage J, Schwab D, Beschorner R, Ebinger M, Schuhmann MU and Schittenhelm J: Analysis of IDH1-R132 mutation, BRAF V600 mutation and KIAA1549-BRAF fusion transcript status in central nervous system tumors supports pediatric tumor classification. J Cancer Res Clin Oncol 142: 89-100, 2016.

21. Gupta K, Orisme W, Harreld JH, Qaddoumi I, Dalton JD, Punchihewa $C$, Collins-Underwood R, Robertson $T$, Tatevossian RG and Ellison DW: Posterior fossa and spinal gangliogliomas form two distinct clinicopathologic and molecular subgroups. Acta Neuropathol Commun 2: 18, 2014

22. Qaddoumi I, Orisme W, Wen J, Santiago T, Gupta K, Dalton JD Tang B, Haupfear K, Punchihewa C, Easton J, et al: Genetic alterations in uncommon low-grade neuroepithelial tumors: BRAF, FGFR1, and MYB mutations occur at high frequency and align with morphology. Acta Neuropathol 131: 833-845, 2016.

23. Ballester LY, Fuller GN, Powell SZ, Sulman EP, Patel KP, Luthra R and Routbort MJ: Retrospective analysis of molecular and immunohistochemical characterization of 381 primary brain tumors. J Neuropathol Exp Neurol 76: 179-188, 2017.
24. Alexandrescu S, Korshunov A, Lai SH, Dabiri S, Patil S, Li R, Shih CS, Bonnin JM, Baker JA, Du E, et al: Epithelioid glioblastomas and anaplastic epithelioid pleomorphic xanthoastrocytomas-same entity or first cousins? Brain Pathol 26: 215-223, 2016.

25. Kleinschmidt-DeMasters BK, Alassiri AH, Birks DK, Newell KL, Moore W and Lillehei KO: Epithelioid versus rhabdoid glioblastomas are distinguished by monosomy 22 and immunohistochemical expression of INI-1 but not claudin 6 . Am J Surg Pathol 34: 341-354, 2010.

26. Tanaka S, Nakada M, Nobusawa S, Suzuki SO, Sabit H, Miyashita K and Hayashi Y: Epithelioid glioblastoma arising from pleomorphic xanthoastrocytoma with the BRAF V600E mutation. Brain Tumor Pathol 31: 172-176, 2014.

27. Broniscer A, Tatevossian RG, Sabin ND, Klimo P Jr, Dalton J, Lee R, Gajjar A and Ellison DW: Clinical, radiological, histological and molecular characteristics of paediatric epithelioid glioblastoma. Neuropathol Appl Neurobiol 40: 327-336, 2014.

28. Dahiya S, Emnett RJ, Haydon DH, Leonard JR, Phillips JJ, Perry A and Gutmann DH: BRAF-V600E mutation in pediatric and adult glioblastoma. Neuro Oncol 16: 318-319, 2014.

29. Takahashi Y, Akahane T, Sawada T, Ikeda H, Tempaku A, Yamauchi S, Nishihara $\mathrm{H}$, Tanaka S, Nitta $\mathrm{K}$ and Ide $\mathrm{W}$, et al: Adult classical glioblastoma with a BRAF V600E mutation. World J Surg Oncol 13: 100, 2015.

30. Sperveslage J, Gierke M, Capper D, Honegger J, Sipos B, Beschorner R and Schittenhelm J: VE1 immunohistochemistry in pituitary adenomas is not associated with BRAF V600E mutation. Acta Neuropathol 125: 911-912, 2013.

31. Chapman PB, Hauschild A, Robert C, Haanen JB, Ascierto P, Larkin J, Dummer R, Garbe C, Testori A, Maio M, et al: Improved survival with vemurafenib in melanoma with BRAF V600E mutation. N Engl J Med 364: 2507-2516, 2011.

32. Sosman JA, Kim KB, Schuchter L, Gonzalez R, Pavlick AC, Weber JS, McArthur GA, Hutson TE, Moschos SJ, Flaherty KT, et al: Survival in BRAF V600-mutant advanced melanoma treated with vemurafenib. N Engl J Med 366: 707-714, 2012.

33. Chamberlain MC: Salvage therapy with BRAF inhibitors for recurrent pleomorphic xanthoastrocytoma: A retrospective case series. J Neurooncol 114: 237-240, 2013.

34. Lee EQ, Ruland S, LeBoeuf NR, Wen PY and Santagata S: Successful treatment of a progressive BRAF V600E-mutated anaplastic pleomorphic xanthoastrocytoma with vemurafenib monotherapy. J Clin Oncol 34: e87-e89, 2016.

35. Robinson GW, Orr BA and Gajjar A: Complete clinical regression of a BRAF V600E-mutant pediatric glioblastoma multiforme after BRAF inhibitor therapy. BMC Cancer 14: 258, 2014.

36. Rush S, Foreman N and Liu A: Brainstem ganglioglioma successfully treated with vemurafenib. J Clin Oncol 31: e159-e160, 2013.

This work is licensed under a Creative Commons Attribution-NonCommercial-NoDerivatives 4.0 International (CC BY-NC-ND 4.0) License. 